\title{
Ethyl acetate extraction from a Chinese herbal formula, Jiedu Xiaozheng Yin, inhibits the proliferation of hepatocellular carcinoma cells via induction of G0/G1 phase arrest in vivo and in vitro
}

\author{
ZHIYUN CAO ${ }^{1}$, WEI LIN $^{1}$, ZHENGRONG HUANG $^{2}$, XUZHENG CHEN $^{1}$, JINYAN ZHAO $^{1}$, \\ LIANGPU ZHENG ${ }^{1}$, HONGZHI YE ${ }^{1}$, ZHIZHEN LIU ${ }^{1}$, LIANMING LIAO ${ }^{1}$ and JIAN DU ${ }^{1}$ \\ ${ }^{1}$ Fujian Academy of Integrative Medicine, Fujian University of Traditional Chinese Medicine; \\ ${ }^{2}$ Integrated Chinese and Western Medicine Department, Fujian Provincial Cancer Hospital, Fuzhou, P.R. China
}

Received September 1, 2012; Accepted October 8, 2012

DOI: 10.3892/ijo.2012.1703

\begin{abstract}
Jiedu Xiaozheng Yin (JXY), a polyherbal formula of traditional Chinese medicine (TCM), has been used to treat various kinds of cancer in China. However, the mechanism of its anticancer activity has yet to be elucidated. Air-dried herbs were extracted with reagents of different polarity. HepG2 cells were treated with different doses of ethyl acetate extract (EE-JXY) and chloroform extract (CE-JXY) for $24 \mathrm{~h}$. Cell viability was detected by MTT assay. Colony formation ability was also evaluated. Cell cycle was evaluated by FACS. Tumor bearing $\mathrm{BALB} / \mathrm{c}$ nude mice was treated with EE-JXY $(0.06 \mathrm{~g} / \mathrm{kg})$ for 20 days. Tumor volume and weight were monitored. The percentage of PCNA-positive cells and the level of G1 phase proteins [cyclin-dependent kinase2 (CDK2), cyclin-dependent kinase4 (CDK4), cyclin D and cyclin E and G2 phase proteins [cyclin-dependent kinase1 (CDK1), cyclin A and cyclin B] were detected by immunohistochemistry and western blotting.
\end{abstract}

Correspondence to: Professor Jian Du or Dr Lianming Liao, Academy of Integrative Medicine, Fujian University of Traditional Chinese Medicine, no. 1 Huatuo Road, Fuzhou, Fujian 350108, P.R. China

E-mail: dujian@fjtcm.edu.cn

E-mail: 1lm@fjtcm.edu.cn

Abbreviations: JXY, Jiedu Xiaozheng Yin; TCM, traditional Chinese medicine; EE-JXY, ethyl acetate fraction; CE-JXY, chloroform fraction; PCNA, proliferating cell nuclear antigen; CDK2, cyclin-dependent kinase2; CDK4, cyclin-dependent kinase4; CDK1, cyclin-dependent kinase1; HCC, hepatocellular carcinoma; HDW, Hedyotis Diffusa Willd; SF, Sophora flavescens; PC, Pseudobulbus Cremastrae; CDKs, cyclin-dependent kinases; DMEM, Dulbecco's modified Eagle's medium; FBS, fetal bovine serum; PE-JXY, ether extract; BE-JXY, n-BuOH extract; TV, tumor volume; TBS, SuperBlock T20; TBST, Tween-20; ANOVA, one-way analysis of variance; $\mathrm{Rb}$, retinoblastoma susceptibility protein

Key words: proliferation, Jiedu Xiaozheng Yin, hepatocellular carcinoma, cell cycle
EE-JXY and CE-JXY dose-dependently inhibited the growth of HepG2 cells ( $\mathrm{P}<0.01$ for both). Furthermore, EE-JXY inhibited the formation of cell colonies and blocked the cell cycle to G1 phase in a dose-dependent manner $(\mathrm{P}<0.01$ for all). EE-JXY showed an obviously antitumor effect in vivo $(\mathrm{P}<0.05)$. Further investigation showed that EE-JXY decreased the proliferation index of tumors $(\mathrm{P}<0.01)$ through increasing the expression of G1-related proteins (cyclin D and cyclin $\mathrm{E}, \mathrm{P}<0.05$ and $\mathrm{P}<0.01$ ). These results suggested that JXY inhibits the growth of HepG2 cells at least via arresting the cell cycle at the G0/G1 phase.

\section{Introduction}

Hepatocellular carcinoma (HCC) is listed as the fifth most common malignancy in the world. Although surgical treatment and non-surgical therapeutic modalities such as chemotherapy, radiotherapy and interventional therapy have been employed, HCC is rarely curative $(1,2)$. For this reason more and more patients and oncologists are seeking alternative medicines to improve the curative rate of HCC. Traditional Chinese medicine (TCM) has been widely used in China to treat HCC because it can improve immune function of patients and alleviate chemoradiotherapy-related side effects (3).

In TCM, herbs commonly used for cancer treatment can be divided into two categories. One is nutritious and tonic herbs and the other is heat-clearing and detoxification herbs. Nutritious and tonic herbs (Fuzheng herbs) act by improving the immune function to fight cancer (such as Astragalus, Ganoderma lucidum and ginseng) (4-6). Heat clearing and detoxication herbs (Qingre Jiedu herbs) may directively kill tumor cells [such as Hedyotis diffusa Willd (HDW), Sophora flavescens (SF), Pseudobulbus Cremastrae (PC), Prunella, Bidens, banzhilian] (7-9). In TCM, cancer is considered to be caused by accumulation of foreign toxins, and cancer itself is also considered to be a toxin that is harmful to human body. The above herbs may contain ingredients that can inhibit proliferation and promote apoptosis of tumor cells. For example, we found that HDW water extract may inhibit proliferation of cancer cells via regulating the cell cycle (10). 
Others reported HDW and Prunella may induce cell apoptosis via the mitochondrion-dependent pathway (11-14).

Cell proliferation is controlled by proteins relating to cell cycle in the cytoplasm. Among them are cyclins and cyclin-dependent kinases (CDKs). Protein phosphorylation by these kinases is the basis of a cascade of signaling that pushes a cell from one stage to the next. A set of checkpoints that monitor completion of critical events is involved $(15,16)$. Kinase activation generally requires association with a second subunit that is transiently expressed at the appropriate period of the cell cycle. A cyclin may associate with a CDK to create an active complex with unique substrate specificity. Regulating of phosphorylation and dephosphorylation of CDK-cyclin complexes ensure the normal transition of cell cycle stages $(17,18)$. Arresting cell cycle in $\mathrm{G} 1$ phase or $\mathrm{G} 2 / \mathrm{M}$ phase is one of the mechanisms used by anticancer medicines $(19,20)$.

JXY is a polyherbal formula of TCM according to the theory of Chinese medicine. It is composed of HDW (30 g), Prunella $(15 \mathrm{~g})$, PC (15 g) and SF (15 g). These herbs are capable of heat-clearing and detoxification. Our previous clinical studies have shown that JXY can prolong the cancer patients' overall survival time and improve the patients' quality of life (21). By using molecular docking simulation, Chen et al showed that some components in HDW, Prunella and PC can combine with CDK2 (22). In the present study, we evaluated the effect of JXY on proliferation of hepatoma cells and its effects on the cell cycle.

\section{Materials and methods}

Reagents. Dulbecco's modified Eagle's medium (DMEM), fetal bovine serum (FBS), penicillin-streptomycin, western blot detection stack/iblot dry blotting system, mouse antibodies against PCNA, CDK1, CDK2, CDK4, cyclin B, cyclin D, cyclin $\mathrm{E}$ and cyclin A were from Maixin Bio (Fuzhou, China). Cycle test ${ }^{\mathrm{TM}}$ plus DNA reagent kit was purchased from Becton-Dickinson (San Jose, CA, USA). All other chemicals, unless otherwise stated, were obtained from Sigma Chemicals (St. Louis, MO, USA).

Preparation of ethyl acetate extract from JXY.JXY is composed of HDW (30 g), Prunella (15 g), PC (15 g) and SF (15 g). Four herbs of JXY were purchased from Guo Yi Tang Hospital of Fujian University of Traditional Chinese Medicine (Fuzhou, China). JXY (7.5 kg) was refluxed with $75 \%$ ethanol for $2 \times 3 \mathrm{~h}$ to obtain total extract (TE-JXY). The alcohol was removed under vacuum using a rotary evaporator. The residue was suspended in water, which was partitioned sequentially with petroleum ether, chloroform, ethyl acetate and $\mathrm{n}-\mathrm{BuOH}$ to afford an petroleum ether extract (PE-JXY), n-BuOH extract (BE-JXY), CE-JXY and EE-JXY. Four extracts were evaporated in vacuum and stored at $4^{\circ} \mathrm{C}$ prior to use. PE-JXY, CE-JXY, EE-JXY and BE-JXY were diluted in DMSO into $200 \mathrm{mg} / \mathrm{ml}$ for in vitro experiments. For in vivo study EE-JXY was dissolved in NS to a final concentration of $6 \mathrm{mg} / \mathrm{ml}$.

Cell culture. Human hepatoma cells lines HepG2 was obtained from American Type Culture Collection (ATCC, Manassas, VA, USA). The cells were grown in DMEM containing $10 \% \mathrm{FBS}$, $100 \mathrm{U} / \mathrm{ml}$ penicillin and $100 \mu \mathrm{g} / \mathrm{ml}$ streptomycin in $37^{\circ} \mathrm{C}$ humidified incubator with $5 \% \mathrm{CO}_{2}$. The cells were subcultured at 80 to $90 \%$ confluence. Cells used in this study were subjected to no more than 20 cell passages.

Tumor xenograft. Sixteen Male BALB/c nude mice with body weight from 18 to $22 \mathrm{~g}$ were injected with HepG2 cells suspension at the right flank. After 7 days mice were randomly divided into two groups (vehicle group and EE-JXY group). EE-JXY $(0.06 \mathrm{~g} / \mathrm{kg})$ was administered twice every day in the EE-JXY group while the same volume of NS was administered for the vehicle group. During treatment tumor size was measured and volume was calculated according to the following formula: tumor volume $\left(\mathrm{TV}, \mathrm{mm}^{3}\right)=\mathrm{d}^{2} \mathrm{xD} / 2$, where $\mathrm{d}$ and $\mathrm{D}$ were the shortest and longest diameter, respectively. On day 21 tumor was excised and weighed. The animals were maintained in a pathogen-free facility $\left(23 \pm 2^{\circ} \mathrm{C}, 55 \pm 5 \%\right.$ humidity). Food and water were provided ad libitum. All procedures on treating mice were performed according to Animal Care Guidelines issued by Ministry of Science and Technology of the People's Republic of China and the Animal Care Committee of Fu Jian University of Traditional Chinese Medicine approved our protocols.

Evaluation of cell viability by MTT assay. Cell viability was evaluated by MTT colorimetric assay; $1 \times 10^{4}$ cells/well were seeded into 96-well plates. The cells were treated with different concentrations of PE-JXY, EE-JXY, BE-JXY or CE-JXY for different times. Then $20 \mu \mathrm{l}$ MTT $(5 \mathrm{mg} / \mathrm{ml})$ was added to each well. After $4 \mathrm{~h}$, MTT was discarded and $100 \mu \mathrm{l}$ DMSO was added to each well. The absorbance was measured at $490 \mathrm{~nm}$ with a microplate reader (Biotek, Winooski, VT, USA). The cell viability was calculated according to the following formula: cell viability $(\%)=$ average absorbance of JXY-containing group/average absorbance of blank group x $100 \%$.

Cell cycle analysis. After incubation with different concentrations of EE-JXY for $24 \mathrm{~h}$, cells were digested with trypsinase and washed twice with PBS. Single cell suspension with a final concentration of $1 \times 10^{6} / \mathrm{ml}$ was prepared. Cell cycle analysis was evaluated with flow cytometry according to the instructions of DNA Plus kit and the percents of G0/G1-phase, S-phase and G2/M-phase were calculated with the ModFit software (BD Biosciences, San Jose, CA, USA).

Soft-agar colony formation assay. After HepG2 cells were treated with EE-JXY for $24 \mathrm{~h}$, cells were harvested and pipetted well to become single-cell suspension in DMEM with 10\% FBS at a concentration of $5 \times 10^{4}$ cells $/ \mathrm{ml}$. Normal melting point agar (0.4 ml of $0.4 \%$ agar in $1.6 \mathrm{ml}$ DMEM containing $10 \% \mathrm{FBS}$ ) was placed into each well of a 6 -well plate. After solidification of the bottom agar, $1 \mathrm{ml}$ of cell mixture consisting of $0.02 \mathrm{ml}$ of cell suspension $\left(5 \times 10^{4}\right.$ cells $\left./ \mathrm{ml}\right)$ and $0.98 \mathrm{ml}$ of $0.8 \%$ lower melting point agar in DMEM containing $10 \%$ FBS were poured over the bottom agar. After solidification of the upper agar wells were incubated at $37^{\circ} \mathrm{C}$ in a humidified $5 \% \mathrm{CO}_{2}$ atmosphere for 1 week. Colony formation in the agar was then photographed and counted under a phase-contrast microscope.

Western blot analysis. Tumor were lysed with lysis buffer (M-PER; Thermo Scientific, Rockford, IL, USA) containing protease and phosphatase inhibitor cocktails (EMD 

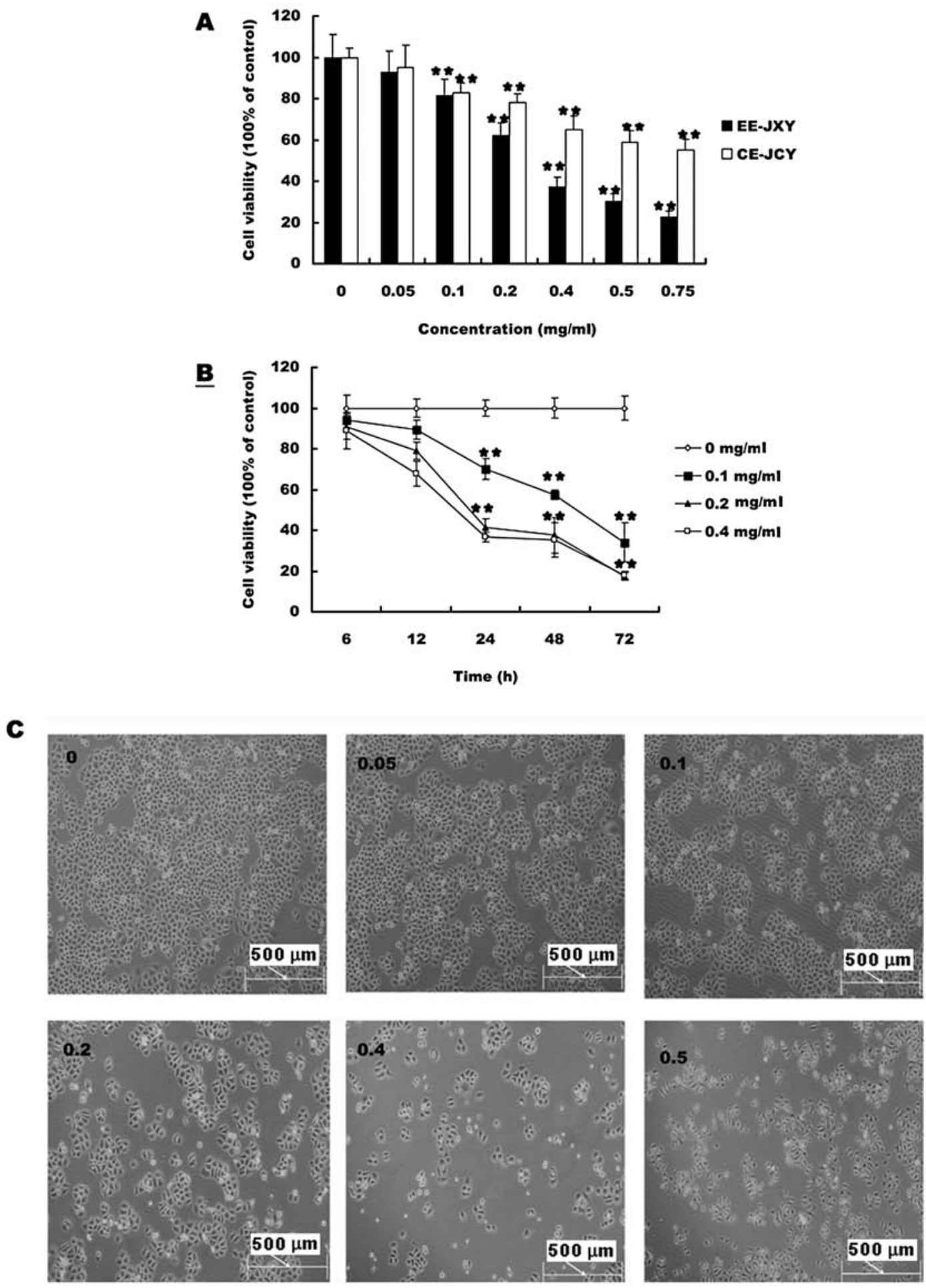

Figure 1. Effect of EE-JXY on HepG2 cells viability. (A) HepG2 cells were treated with the indicated concentrations of EE-JXY and CE-JXY for 24 h. (B) Cells were treated with indicated concentrations of EE-JXY for the indicated times. The data were normalized to the viability of control cells $(100 \%$, treated with $0.5 \%$ DMSO). Data are mean $\pm \mathrm{SD} .{ }^{* *} \mathrm{P}<0.01$, vs control cells. (C) Cells were treated with the indicated concentrations of EE-JXY for $24 \mathrm{~h}$ and confluent changes were observed using phase-contrast microscopy. The photographs were taken at a magnification, x100. Images are representative of three independent experiments.

Biosciences La Jolla, CA, USA and Sigma Chemical, respectively). The lysates were resolved in $12 \%$ SDS-PAGE gels and electroblotted using the iblot western detection stack/iblot dry blotting system. The PVDF membranes were blocked with SuperBlock T20 (TBS) blocking buffer (Thermo Scientific) for $30 \mathrm{~min}$ and washed in TBS with 0.25\% Tween-20 (TBST), followed by incubation overnight at $4^{\circ} \mathrm{C}$ with primary antibody. After washing with TBST, the membranes were incubated with secondary antibody for $1 \mathrm{~h}$. The membranes were developed using Super Signal Pico Substrate (Thermo Scientific), and images were taken using a Kodak Image Station 400R (Kodak, Rochester, NY, USA). 

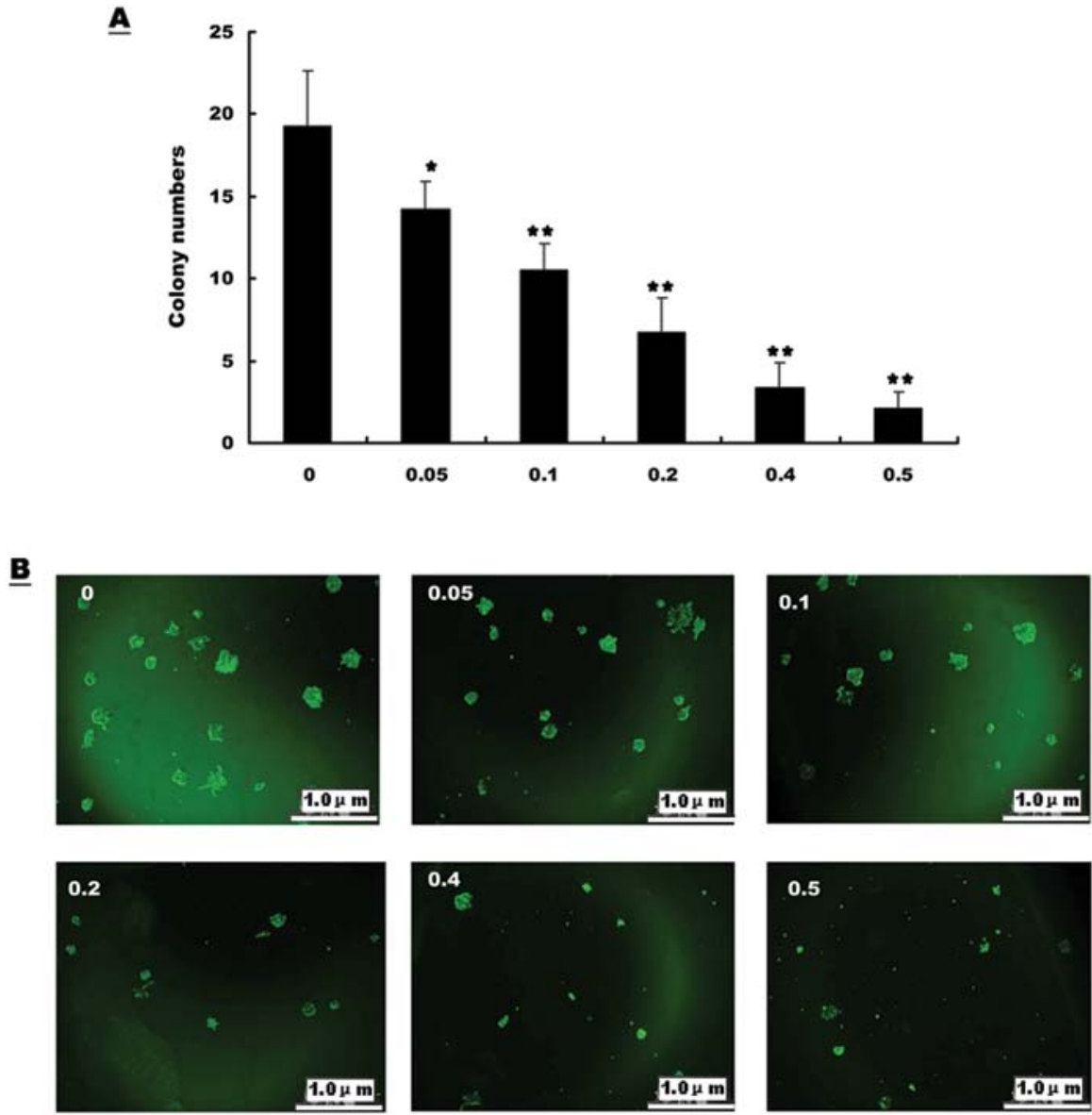

Figure 2. Effect of EE-JXY on colony formation of HepG2 cells. (A) HepG2 cells were pretreated with EE-JXY at indicated concentrations for $24 \mathrm{~h}$ and then $1 \times 10^{3}$ single cells were plated on agar to form colonies. Graphs represent averages of three independent experiments. Data are mean \pm SD. ${ }^{*} \mathrm{P}<0.05$, ${ }^{* *} \mathrm{P}<0.01 \mathrm{vs}$ control cells. (B) A representative picture is shown.

Immunohistochemistry assay. After fixing in $10 \%$ formalin buffer for $24 \mathrm{~h}$, tumor samples were processed in a routine method for paraffin-embedded tumor sections. Sections were subjected to antigen retrieval and blocking of endogenous peroxidase activity. For immunostaining, sections were incubated with the primary antibodies (mouse monoclonal anti-PCNA (1:150; Maixin Bio), mouse monoclonal anti-CDK1, anti-CDK2, anti-CDK4 (1:150; 1:100, 1:150, Maixin Bio), mouse monoclonal anti-cyclin $\mathrm{D}$, anti-cyclin $\mathrm{E}$, anti-cyclin $\mathrm{A}$ and anti-cyclin B (1:100; 1:150, 1:150, 1:100, Maixin Bio). Sections were then incubated with biotinylated appropriate secondary antibody followed by conjugated horseradish peroxidase (HRP)streptavidin (Maixin Bio). Then 3,3'-diaminobenzidine (DAB; Sigma Chemicals) was added, incubated at room temperature and counterstaining with diluted Harris hematoxylin (Sigma Chemicals). Cells were quantified by counting positive cells and total number of cells at five arbitrarily selected fields from each tumor at magnifications, $\mathrm{x} 100$. Data are presented as percentage of positive cells.

Statistical analysis. Data are shown as mean values \pm SD and analyzed using SPSS package for Windows (Version 11.5). Statistical analysis was performed with the Student's t-test and one way analysis of variance (ANOVA). Dunnett's was used as post hoc test. Differences at $\mathrm{P}<0.05$ were considered statistically significant.

\section{Results}

Effects of JXY extracts on the growth of Hep 22 cells. The effects of PE-JXY, BE-JXY, CE-JXY and EE-JXY on the growth of HepG2 cells was detected by MTT assay. As shown in Fig. 1A cell viability was inhibited by both CE-JXY and EE-JXY in a dose-dependent manner. Treatment with 0.05 to $0.75 \mathrm{mg} / \mathrm{ml}$ of CE-JXY and EE-JXY for $24 \mathrm{~h}$ reduced cell viability by 5 to $40 \%$ and 9 to $75 \%$, respectively, compared to untreated control cells $(\mathrm{P}<0.01$ for both). Greater inhibition was observed for EE-JXY than for CE-JXY at the same concentration. Inhibition of cell viability by PE-JXY or BE-JXY was weak (data not shown). We also evaluated the effect of 0.1, 0.2 and $0.4 \mathrm{mg} / \mathrm{ml}$ of EE-JXY on cell viability with different culture time. As shown in Fig. 1B and C, cell viability was inhibited by EE-JXY in a time-dependent manner.

EE-JXY inhibits colony formation ability of tumor cells. In order to determine the effect of EE-JXY on colony formation of HepG2, cells treated with different concentrations of EE-JXY were cultured in semisolid culture media for 1 week followed by manual counting of colonies. The results showed that as the concentration of EE-JXY increased, the colony size and the colony number decreased (Fig. 2A). Thus the colony formation ability of HepG2 cells was significantly inhibited by EE-JXY (Fig. 2B). 
A

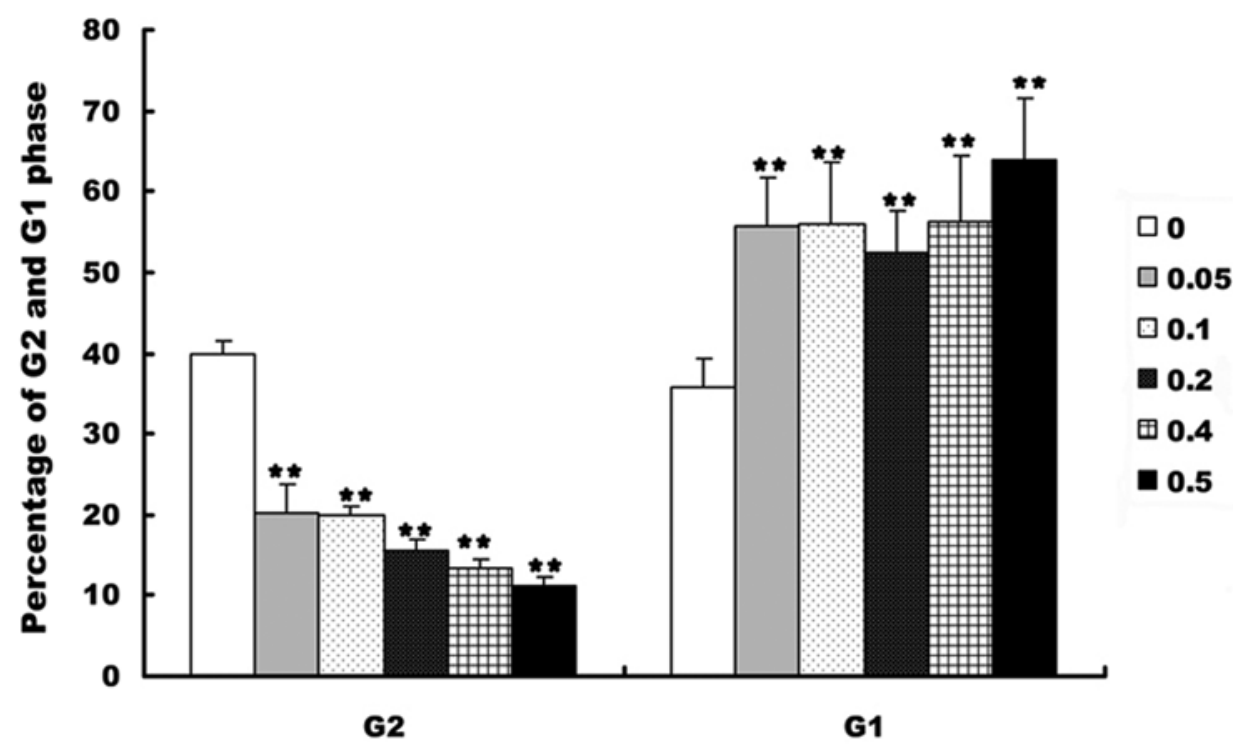

B
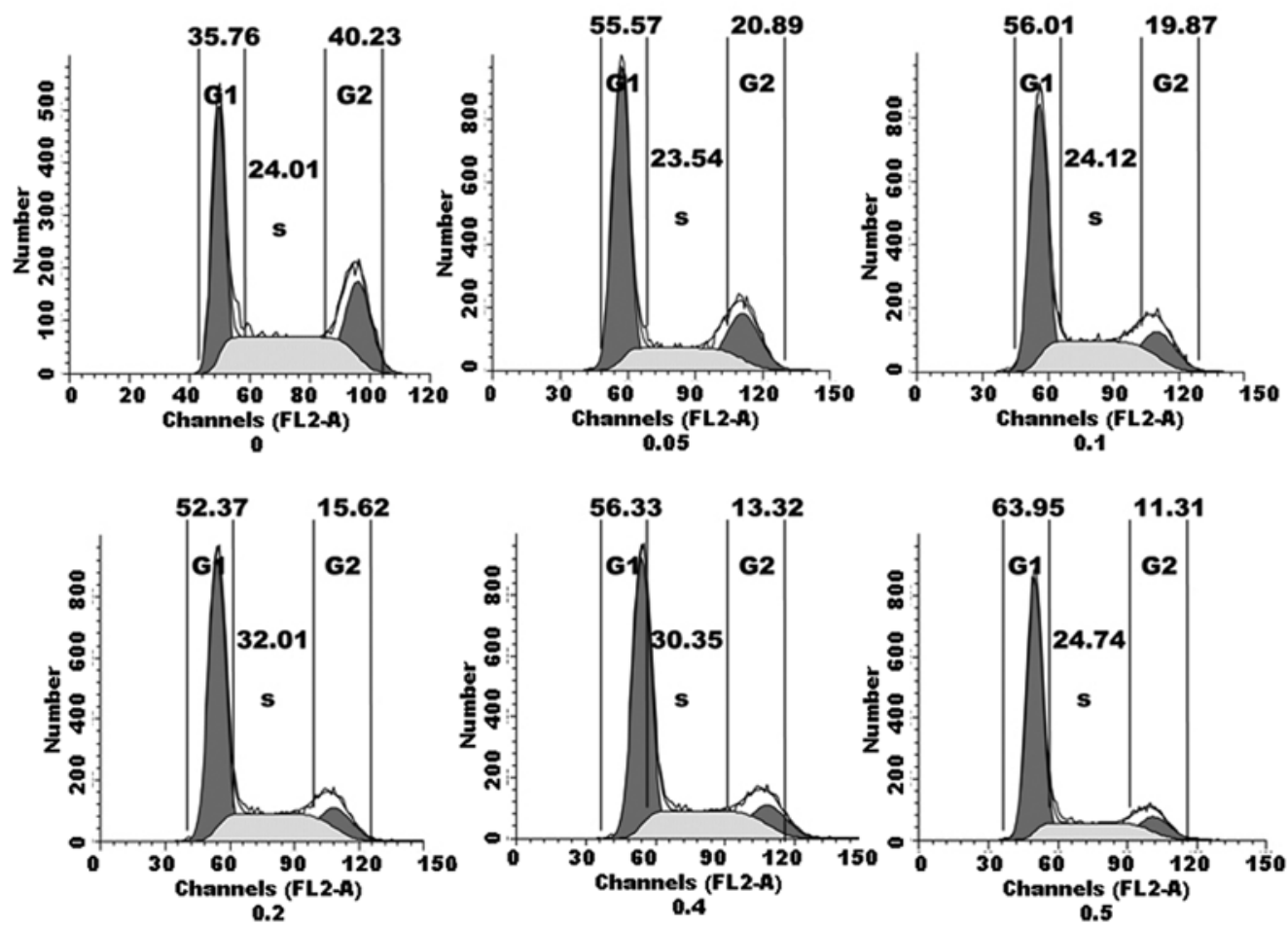

Figure 3. Effect of EE-JXY on HepG2 cell cycle progression. (A) Cells were treated with the indicated concentrations of EE-JXY for 24 h, stained with PI and Annexin $\mathrm{V}$ and analyzed by flow cytometry. The proportion of cells in each phase of the cell cycle was calculated using ModfitLT Version 3.0 Software. The percentages of cells in G2 and G1 phase for each concentration of EE-JXY was compared. Data shown are mean \pm SD. ${ }^{* *} \mathrm{P}<0.01$ vs control cells (B) Representative assays are shown for each concentration of EE-JXY.

EE-JXY induce cell cycle arrest of HepG2 cells. We investigated the cell cycle after HepG2 cells were treated with different concentrations of EE-JXY for $24 \mathrm{~h}$. The results showed that EE-JXY markedly increased the percentage of cells in G1 phase (Fig. 3A, P<0.01) and decreased the percentage of cells in $\mathrm{G} 2$ phase in a dose-dependent manner (Fig. 3A, P<0.01). These results demonstrated that EE-JXY induced cell cycle arrest of HepG2 cells. The representative results of cell cycle are shown in Fig. 3B.
EE-JXY inhibits tumor growth in vivo. After mice were treated for 20 days, tumor volume was reduced by $39 \%(\mathrm{P}<0.05)$ in EE-JXY group compared with vehicle group (Fig. 4A). A comparison of tumor weight between EE-JXY group and vehicle group showed a similar tendency (Fig. 4B, $\mathrm{P}<0.05$ ) with tumor volume. More importantly, the body weights between vehicle group and EE-JXY group was similar at the end of treatment, indicating EE-JXY is safe (Fig. 4C). 
A

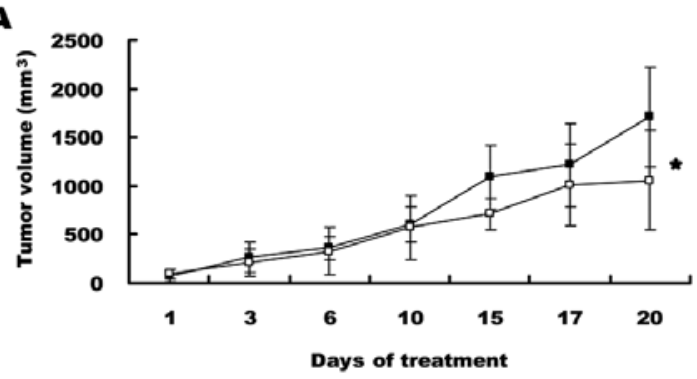

C

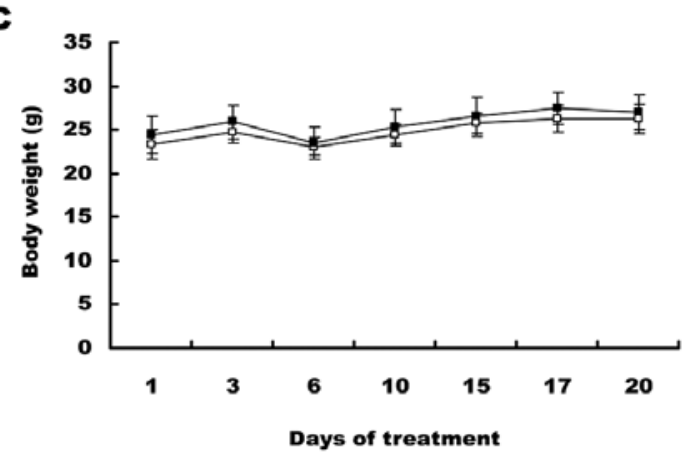

- - Vehicle

$\rightarrow-$ EE-JXY

- Vehicle

$\rightarrow-E E-J X Y$

A

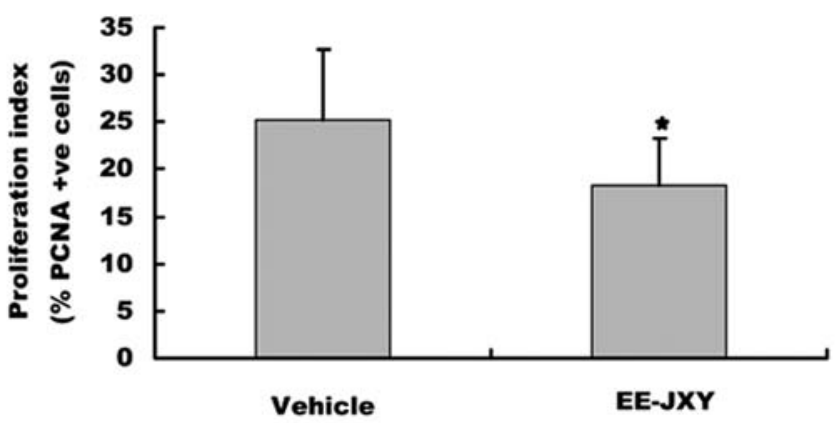

B
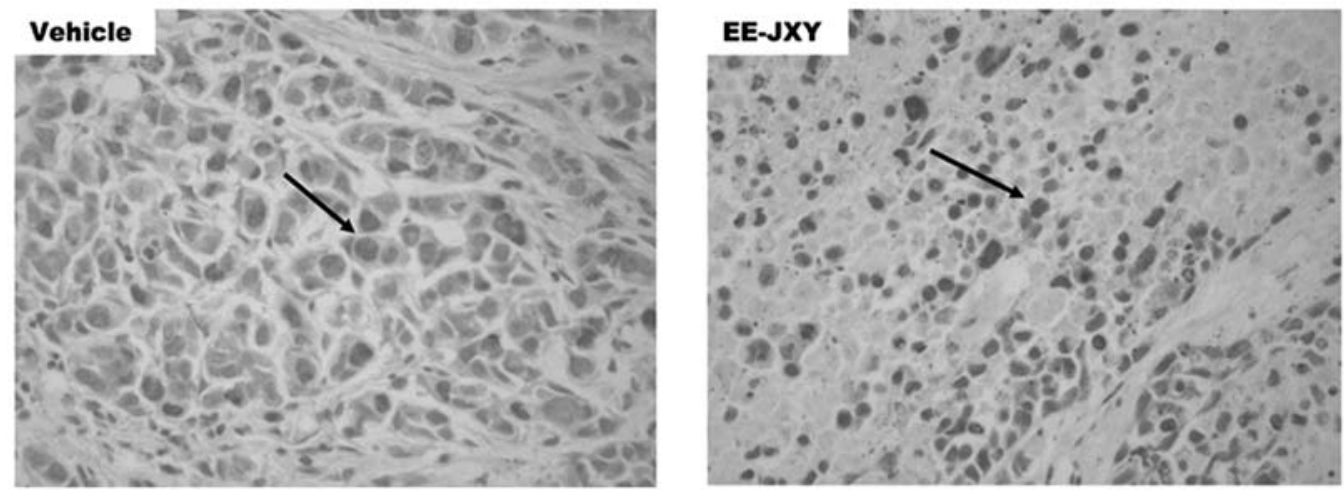

Figure 5. Effects of EE-JXY on proliferation index of tumor. (A) Tumor bearing BALB/c nude mice. were treated with either EE-JXY $(0.06 \mathrm{~g} / \mathrm{kg})$ or saline for 20 days as described in Materials and methods. Then tumor tissue was stained for PCNA. Proliferation index is shown as percentage of PCNA-positive cells. Values are mean $\pm \mathrm{SD}, \mathrm{n}=6,{ }^{\prime \prime} \mathrm{P}<0.05$ vs control group. (B) Representative images of immunohistochemical staining for control group and EE-JXY group are shown. Positive cells are indicated by arrows. Magnification, x100.

The effect of EE-JXY on proliferation of tumor. We also detected the proliferation index of tumor after mice treated with EE-JXY for 20 days by immunohistochemical staining assay. The results showed that the proliferation index of EE-JXY group was significantly lower than the vehicle group (Fig. 5A,

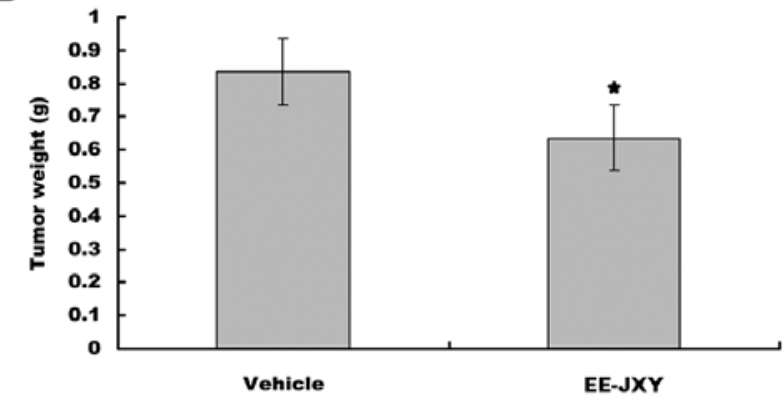

Figure 4. Effect of EE-JXY on tumor growth. Tumor bearing BALB/c nude mice. were treated with either EE-JXY $(0.06 \mathrm{~g} / \mathrm{kg})$ or saline for 20 days as described in Materials and methods. Then final (A) tumor volume and (B) tumor weight were measured. (C) The body weight was weighed every 3 days. Values are mean $\pm \mathrm{SD}, \mathrm{n}=8,{ }^{*} \mathrm{P}<0.05$ vs control group. 
A


C
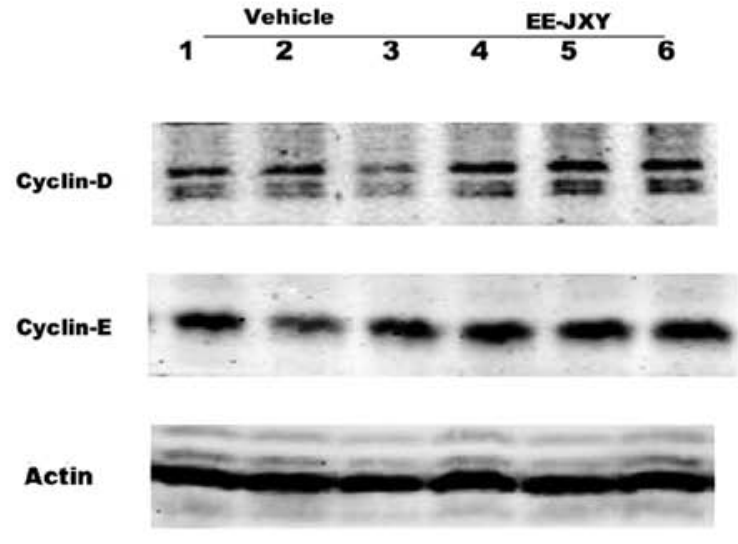

Figure 6. Effects of EE-JXY on cyclin D and cyclin E. (A) Tumor-bearing BALB/c nude mice were treated with either EE-JXY (0.06 g/kg) or saline for $20 \mathrm{days}$ as described in Materials and methods. Then tumor tissue was stained for CDK2, CDK4, cyclin D and cyclin E and the results are shown as percentages of the positive cells. Values are mean $\pm \mathrm{SD}, \mathrm{n}=6 .{ }^{.} \mathrm{P}<0.05,{ }^{* *} \mathrm{P}<0.01$ vs control group. (B) Representative photos of immunohistochemical staining for cyclin $\mathrm{D}$ and cyclin $\mathrm{E}$ are shown. Positive cells were indicated by arrow. Magnification, x100. (C) Expression of cyclin D and cyclin E in tumor were further determined by western blot analysis. Actin was used as the internal controls. The photos are representative results of three experiments.

CDK4, cyclin D and cyclin E) in the tumor tissue with immunohistochemical staining and western blot analysis. Immunochemistry showed there were more cells positive for
CDK2 and CDK4 in the EE-JXY group than in the vehicle group, but the difference was not significant (Fig. 6B). There were significantly more cells positive for cyclin D and cyclin E 
in the EE-JXY group than in the vehicle group (Fig. 6A, $\mathrm{P}<0.05$ and $\mathrm{P}<0.01)$. The results were further confirmed by western blot analysis (Fig. 6C).

Next we detected the expression of $\mathrm{G} 2$ phase related proteins (CDK1, cyclin A and cyclin B). Immunohistochemical staining showed that the percentages of cells positive for CDK1, cyclin A or cyclin B were similar between the EE-JXY group and vehicle group (data not shown). These results were confirmed by western blot analysis (data not shown).

\section{Discussion}

According to TCM theory, toxins are one of common causes of cancer. Toxins are referred to as 'fire toxins' and 'heat' which, when accumulating in liver, may lead to liver qi stagnation. In Chinese medicine, a variety of symptoms associated with menopause, depression, headaches, insomnia and fibromyalgia are attributed to liver qi stagnation (23). Toxins can also cause blood stasis. Toxins and heat accumulating in organs will cause cancer. Several herbs are commonly used in China to treat cancer by clearing heat and expelling toxin. These herbs include HDW, Selaginellae doederleinii, Sophora Tonkinensis, Rhizoma Curcumae Ezhu, Prunella, SF and PC (4,24). Pharmacological studies have shown that these herbal medicines have antitumor effect via inducing cell apoptosis, inhibiting cell proliferation and angiogenesis (25-27). JXY is composed of HWD, Prunella, SF and PC. It is used to treat abscesses, ulcerations, swellings, sore throat, bronchitis, tonsillitis, damp heat jaundice (infectious hepatitis) and malignant tumors of liver, lung and stomach, which are all considered to be caused by excess of heat, fire and toxin in the tissues or organs. Our results showed that JXY extracts, especially EE-JXY, can decrease cell viability through arresting cells in G0/G1 phase in vitro and inhibit tumor growth by regulating the level of G1 phase related proteins in vivo.

Cyclins and CDKs are two key classes of cell cycle regulatory proteins that determine a cell's progress through cell cycle (28). Cyclin D is the first cyclin produced in response to extracellular signals (e.g. growth factors). Cyclin D binds to existing CDK4 to form the active cyclin D-CDK4 complex and then phosphorylates the retinoblastoma susceptibility protein $(\mathrm{Rb})(29)$ to activate E2F. Activation of E2F results in transcription of various genes such as cyclin E, cyclin A, DNA polymerase, and thymidine kinase. Cyclin E binds to CDK2, forming the cyclin E-CDK2 complex, which pushes the cell from G1 to $S$ phase (G1/S transition). Our results showed that EE-JXY increased the level of cyclin D and cyclin E, which may be responsible on its effects on cell cycle arrest in G1 phase.

Studies have shown that the HDW and Prunella may inhibit the proliferation of tumor cells by regulating expression of cell cycle-related proteins $(14,30)$. Similarly we found water extract of HWD can arrest the HepG2 cells in G0/G1 phase via downregulating the mRNA and protein levels of CDK2, CDK4 and E2F (10). Matrine, a component rich in SF, inhibited cell proliferation through arresting cells in G0/G1 phase in gallbladder cancer cells (31), prostate cancer cells (32) and hepatoma cells (33). Qin et al and Zhao et al reported that matrine downregulated the expression of cyclin D1 to inhibit the growth of retinoblastoma cells $(34,35)$. The results of the present study with EE-JXY are consistent with these reports, although the underlying mechanism is unknown.

In p53-dependent G1-arrest signal transduction pathway, DNA damage signalling stabilizes the p53 and then induces expression of many target genes. The gene encoding p21 constitutes a crucial target which can bind the cyclin E/CDK2 complex and inhibits the complex activation, as its upregulation leads to G1-arrest following DNA damage (36). In the p53-independent G1-arrest signal transduction pathway, chemokines can ubiquitinate and degradate cell division cycle 25 homolog A that is required for activation of cyclin E/CDK2 complex to inhibit cell proliferation $(37,38)$. Whether EE-JXY actives the p53-dependent or -independent G1-arrest signal transduction pathway to inhibit tumor growth requires further study.

In summary, EE-JXY significantly inhibited the growth of hepatocellular carcinoma in vivo without any toxic effects. The anti-proliferation effect of EE-JXY regulates the expression of cyclin D and cyclin E to arrest cells in G0/G1 phase. The mechanism by which JXY regulates expression of cyclin D and cyclin E need further investigation.

\section{Acknowledgements}

This study was supported by CHEN Ke-ji Integrative Medicine Development Fund (CKJ2010020); International Science Joint Project of the Ministry of Science and Technology of China (2008DFA32200); Key Project of Fujian Province Department of Science and Technology (2008KJB-01); Fujian Province Natural Science Foundation (2010J01197, 2012J01393). National Natural Science Foundation of China (81102582).

\section{References}

1. Llovet JM, Burroughs A and Bruix J: Hepatocellular carcinoma. Lancet 362: 1907-1917, 2003.

2. Gish RG and Baron A: Hepatocellular carcinoma (HCC): current and evolving therapies. IDrugs 11: 198-203, 2008.

3. Konkimalla VB and Efferth T: Evidence-based Chinese medicine for cancer therapy. J Ethnopharmacol 116: 207-210, 2008.

4. Beinfield $\mathrm{H}$ and Korngold E: Chinese medicine and cancer care. Altern Ther Health Med 9: 38-52, 2003.

5. Huang CF, Lin SS, Liao PH, Young SC and Yang CC: The immunopharmaceutical effects and mechanisms of herb medicine. Cell Mol Immunol 5: 23-31, 2008.

6. Pan B, Cheng T, Nan KJ, Qiu GQ and Sun XC: Effect of Fuzheng Yiliu decoction combined with chemotherapy on patients with intermediate and late stage gastrointestinal cancer. World J Gastroenterol 11: 439-442, 2005.

7. Yan Y, Cook J, McQuillan J, Zhang G, Hitzman CJ, Wang Y, Wiedmann TS and You M: Chemopreventive effect of aerosolized polyphenon $\mathrm{E}$ on lung tumorigenesis in $\mathrm{A} / \mathrm{J}$ mice. Neoplasia 9: 401-405, 2007.

8. Fang Y, Zhang Y, Chen M, Zheng $\mathrm{H}$ and Zhang K: The active component of Hedyotis diffusa Willd. Chinese Trad Plant Med 26: 577-579, 2004.

9. Wu YG and Song LR: Shanghai Science and Technology Press. Zhong hua ben cao (Chin) 25: 530-533, 1998.

10. Chen XZ, Cao ZY, Chen TS, Zhang YQ, Liu ZZ, Su YT, Liao LM and Du J: Water extract of Hedyotis Diffusa Willd suppresses proliferation of human HepG2 cells and potentiates the anticancer efficacy of low-dose 5 -fluorouracil by inhibiting the CDK2-E2F1 pathway. Oncol Rep 28: 742-748, 2012.

11. Lin J, Chen Y, Wei L, Chen X, Xu W, Hong Z, Sferra TJ and Peng J: Hedyotis Diffusa Willd extract induces apoptosis via activation of the mitochondrion-dependent pathway in human colon carcinoma cells. Int J Oncol 37: 1331-1338, 2010.

12. Lin J, Wei L, Xu W, Hong Z, Liu X and Peng J: Effect of Hedyotis Diffusa Willd extract on tumor angiogenesis. Mol Med Rep 4: 1283-1288, 2011. 
13. Jitka P, Milan K and Jaromír S: Biological activities of Prunella vulgaris extract. Phytother Res 17: 1082-1087, 2003.

14. Psotova J, Svobodova A, Kolarova H and Walterova D Photoprotective properties of Prunella vulgaris and rosmarinic acid on human keratinocytes. J Photochem Photobiol B 84: 167-174, 2006.

15. Hunter T and Pines J: Cyclins and cancer. II: Cyclin D and CDK inhibitors come of age. Cell 79: 573-582, 1994.

16. Morgan DO: Principles of CDK regulation. Nature 374: 131-134, 1995.

17. Cobrinik D: Pocket proteins and cell cycle control. Oncogene 24: 2796-2809, 2005.

18. Glotzer M: The molecular requirements for cytokinesis. Science 307: 1735-1739, 2005.

19. Tin AS, Sundar SN, Tran KQ, Park AH, Poindexter KM and Firestone GL: Antiproliferative effects of artemisinin on human breast cancer cells requires the downregulated expression of the E2F1 transcription factor and loss of E2F1-target cell cycle genes. Anticancer Drugs 23: 370-379, 2012.

20. Fang XM, Liu B, Liu YB, Wang JJ, Wen JK, Li BH and Han M: Acetylbritannilactone suppresses growth via upregulation of krüppel-like transcription factor 4 expression in HT-29 colorectal cancer cells. Oncol Rep 26: 1181-1187, 2011.

21. Chen LW, Lin J, Chen W and Zhang W: Effect of Chinese herbal medicine on patients with primary hepatic carcinoma in III stage during perioperational period: a report of 42 cases. Zhongguo Zhong Xi Yi Jie He Za Zhi 25: 832-834, 2005 (In Chinese).

22. Chen L, Zheng C and Du J: Study on antitumor mechanism of Qingre Xiaozheng drink by molecular docking method. Clin Pharmacol Ther (Chin) 12: 324-328, 2007.

23. Li ZQ: Traditional Chinese medicine for primary liver cancer World J Gastroenterol 4: 360-364, 1998.

24. Qui JX and Yang JK: Clinical observation and experimental research in treatment of liver cancer at advanced stage by traditional medication of strengthening the Spleen, replenishing Qi clearing away heat and toxic material. J Integrated Trad Chin West Med 7: 275-277, 1987.

25. Yadav SK and Lee SC: Evidence for Oldenlandia diffusa-evoked cancer cell apoptosis through superoxide burst and caspase activation. Zhong Xi Yi Jie He Xue Bao 4: 485-489, 2006.

26. Rasul A, Yu B, Yang LF, Ali M, Khan M, Ma T and Yang H: Induction of mitochondria-mediated apoptosis in human gastric adenocarcinoma SGC-7901 cells by kuraridin and Nor-kurarinone isolated from Sophora flavescens. Asian Pac J Cancer Prev 12: 2499-2504, 2011.
27. Lin CC and Namba T: Historical and herbological studies on the traditional Japanese and Chinese crude drugs. On the 'Shāncí-gū'. Yakushigaku Zasshi 20: 88-98, 1985 (In Japanese).

28. Nigg EA: Cyclin-dependent protein kinases: key regulators of the eukaryotic cell cycle. Bioessays 17: 471-480, 1995.

29. Orlando DA, Lin CY, Bernard A, Wang JY, Socolar JES, Iversen ES, Hartemink AJ and Haase SB: Global control of cellcycle transcription by coupled CDK and network oscillators. Nature 453: 944-947, 2008

30. Feng L, Jia XB, Shi F and Chen Y: Identification of two polysaccharides from Prunella vulgaris $L$. and evaluation on their anti-lung adenocarcinoma activity. Molecules 15: 5093-5103, 2010.

31. Kang SC, Lee CM, Choi H, Lee JH, Oh JS, Kwak JH, Lee JH, Oh JS, Kwak JH and Zee OP: Evaluation of oriental medicinal herbs for estrogenic and antiproliferative activities. Phytother Res 20: 1017-1019, 2006.

32. Zhang Z, Wang X, Wu W, Wang J, Wang Y, Wu X, Fei X, Li S, Zhang J, Dong P, Gu J and Liu Y: Effects of matrine on proliferation and apoptosis in gallbladder carcinoma cells (GBC-SD). Phytother Res 26: 932-937, 2012.

33. Zhang P, Wang Z, Chong T and Ji Z: Matrine inhibits proliferation and induces apoptosis of the androgen-independent prostate cancer cell line PC-3. Mol Med Rep 5: 783-787, 2012.

34. Qin XG, Hua Z, Shuang W, Wang YH and Cui YD: Effects of matrine on HepG2 cell proliferation and expression of tumor relevant proteins in vitro. Pharm Biol 48: 275-281, 2010.

35. Zhao B, Li B, Bai S, Shen L, Ren R, Jonas JB, Xu X, Lu Q and Liu Q: Effects of matrine on proliferation and apoptosis of cultured retinoblastoma cells. Graefes Arch Clin Exp Ophthalmol 250: 897-905, 2012

36. Agami R and Bernards R: Convergence of mitogenic and DNA damage signaling in the G1 phase of the cell cycle. Cancer Lett 177: 111-118, 2002.

37. Brew CT, Aronchik I, Hsu JC, Sheen JH, Dickson RB, Bjeldanes LF and Firestone GL: Indole-3-carbinol activates the ATM signaling pathway independent of DNA damage to stabilize p53 and induce G1 arrest of human mammary epithelial cells. Int J Cancer 118: 857-868, 2006.

38. Alpan RS and Pardee AB: p21WAF1/CIP1/SDI 1 is elevated through a p53-independent pathway by mimosine. Cell Growth Differ 7: 893-901, 1996. 